REPRINT. In: Computer Recognition Systems: Proc. Int. Conf. CORES 2005, pages 363-370, Rydzyna, Poland, May 22-25, 2005. Vol. in series:

Advances in Soft Computing, Springer Verlag. ISBN 3-540-25054-9.

The copyright owner of the paper is Springer-Verlag. doi:doi:10.1007/3-540-32390-2_42

\title{
Scale and Rotation Invariance \\ of the Evidence Accumulation-Based Line Detection Algorithm
}

\author{
Leszek J Chmielewski \\ Institute of Fundamental Technological Research, PAS, Świętokrzyska 21, \\ 00-049 Warsaw, Poland lchmiel@ippt.gov.pl
}

Summary. The scale and rotation invariance properties of a recently proposed algorithm, using the fuzzy evidence accumulation principle, for finding lines (ridges) of non-parametric shapes is analysed. The proposed modifications consist in scaling the accumulated value with the inverse of the line width and further fuzzifying the accumulation process - along the line width. Good invariance properties received are tested on artificial images and confirmed on real-life mammographic images.

\section{Introduction}

The algorithm analysed in the present paper has been recently proposed in [1] and is described in detail in [2]. Two properties of this algorithm will be investigated: its sensitivity to the scale and to the direction of the detected objects. It is assumed that a multiscale line detector which might be described as good should detect narrow lines as well as wide ones, and lines inclined at arbitrary angles, and that the only feature which should differentiate between strong and weak lines should be their contrast with respect to the background.

The algorithm has originally been designed with the application to the analysis of elongated objects in mammographic images. This demanding task (see [3]) necessitates for superior properties of the algorithm, if the decisions made on the grounds of its results are expected to be reliable.

\section{Scale invariance}

In the previous papers [1, 2], the line intensity was primarily defined using the vector sum of tangents to the line at its two edges (see [2], in the same volume, Eq. (3)):

$$
l=c_{d} c_{e}|\boldsymbol{V}| .
$$

The coefficients - directional consistence $c_{d}$ and edgeness consistence $c_{e}$ of the tangent vectors - have been defined in [2]. 
a

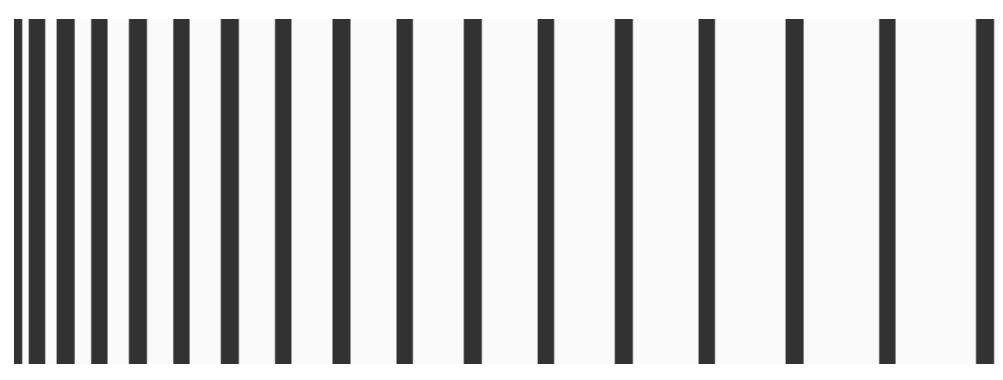

b

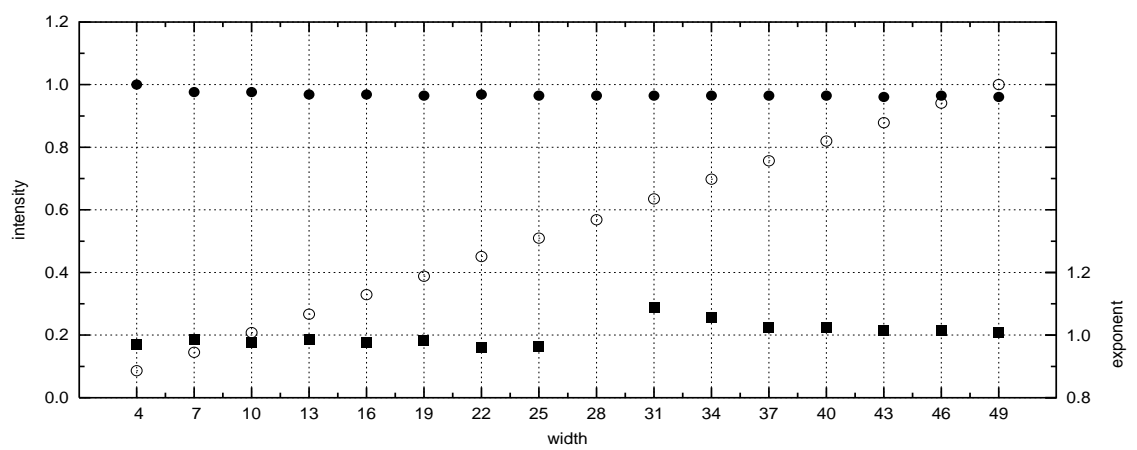

Fig. 1. Introducing scale invariance. (a) test image; (b) graphs of the accumulated value in centres of lines: lineness $l$ according to Eq. (1) (empty circles) and scale invariant lineness $l_{s}$ according to (5) (full circles); exponents found from (4) for subsequent widths (full squares, except reference 28) are all close to one.

We shall understand the scale invariance of the line detector as the independence of the line intensity of the line width. Let us denote such scale invariant line intensity as $l_{s}$. Assume the following relation of this intensity to $l$ as defined in Eq. (1):

$$
l_{s}=l / w^{p} .
$$

The exponent function is introduced to allow for possible non-linearities, potentially stemming from the relation of pixel size to the line width. Let us check for which exponent $p$ the intensity $l_{s}$ is actually invariant to the line width. Let $w_{1}$ and $w_{2}$ be the widths of two lines. It should be then $l_{s}\left(w_{1}\right)=l_{s}\left(w_{2}\right)$ which gives

$$
l_{1} / w_{1}^{p}=l_{2} / w_{2}^{p} .
$$

A test image containing 16 bright lines of width from 4 to 49 pixels every 3 pixels (Fig. 1a) was used to find the exponent $p$. Intensities were taken in maxima at line centres, in the middle row of the image. Line of width 28 pixels was used as a reference and 15 values of $p$ were calculated from

$$
p_{i}=\frac{\ln \left(l_{i} / l_{28}\right)}{\ln \left(w_{i} / w_{28}\right)}, \quad i=4,7, \ldots, 22,25,31,34, \ldots, 49 .
$$


In the graph of $p_{i}$ in Fig. $1 \mathbf{b}$ (squares) it can be seen that all the exponents are close to one, which leads to a simple equation for the scale-invariant accumulated line intensity:

$$
l_{s}=c_{d} c_{e}|\boldsymbol{V}| / w .
$$

In the graph of $p_{i}$ in Fig. $1 \mathbf{b}$ (full circles) it can be seen that the line intensity according to this equation is indeed close to constant for all the widths.

An example of results for a real-life image is shown in Fig. 2. The input image is a window $300 \times 300$ starting from pixel $(850,170)$ of the mammographic image MDB007LL.TIF from the MIAS database [4], originally $4320 \times 2400$. Calculations were made for line widths from 7 to 35 and lineness threshold coefficient 0.25 . Time of calculations was 23 min (Pentium $1 \mathrm{Ghz}$ ).

a

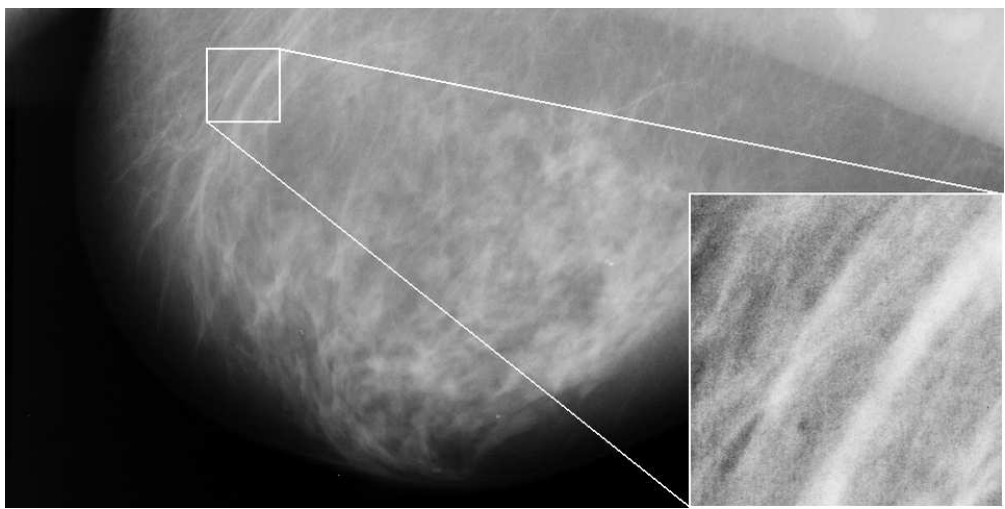

b

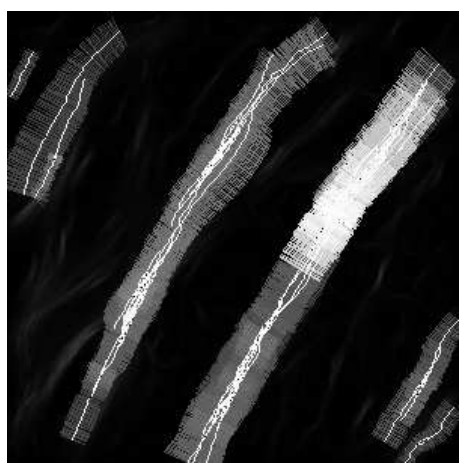

c

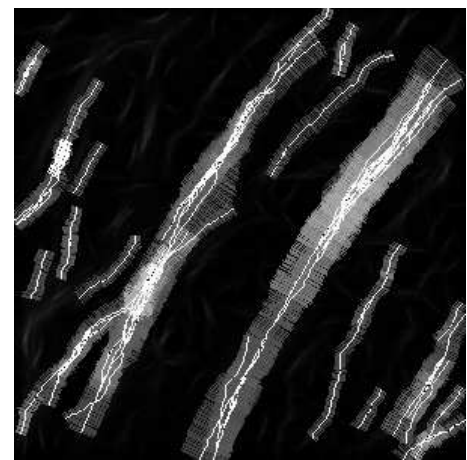

Fig. 2. Example of results for a mammographic image. (a) input: window of image MDB007LL.TIF from the MIAS database; (b) accumulated lineness according to Eq. (1) with lineness ridges and line widths - overestimated line widths and narrow lines overlooked; (c) accumulated scale-invariant lineness according to Eq. (5) with lineness ridges and line widths - the number of relevant details is larger. 


\section{Rotation invariance}

The rotation invariance was tested on seven series of images containing lines of various inclination and widths. Experiments on four series of test images, sufficiently demonstrating the phenomenon of interest, will be reported. In each series there were images of a line 11 pix wide, inclined at angles growing from 0 to $90^{\circ}$ by $5^{\circ}$, and by $0.1^{\circ}$ near $45^{\circ}$ (Fig. 3). Line brightness was 250 and background was 50. Each of the series corresponds to a class of typical line edges which can appear in an image: a line plotted with standard antialiasing, possibly with noise (additive, Gaussian); a line without anti-aliasing, and a line with blurred edges in the form of gentle slopes. The size of the images was $127 \times 127$ pixels, so that the line was narrow with respect to the image. For images with the line at subsequent angles, the line intensity according to Eq. (5) and the line width in the central pixel $(63,63)$ were recorded.

The results are shown in Fig. 4. For easier comparisons, the relative line intensity in the graphs is plotted, with the unit corresponding to 120000. For all the cases, the discrepancies of the lineness from the constant function are substantial, except for the normally anti-aliased lines with noise. The errors of width, which should be 11 in all the cases, are moderate to large, except for the normally anti-aliased lines.

The largest discrepancies appeared at 'round' angles: $0^{\circ}, 45^{\circ}$ and $90^{\circ}$. This phenomenon can be investigated by visualizing the influences of specific pixels on the line intensity. For angle $44.2^{\circ}$ (Fig. 5) some pixels have much smaller influence than the others, which results in smaller line intensity. This is due to that the rate of errors made in the calculation of width at angles close to the 'round' angles is much different from that for other angles (Fig. 6).

The erroneous calculations are closely related to the square tessellation of the image. The angular distribution of image properties is far from isotropic, which can not be avoided. However, the unevenness of the distribution of errors across the edge widths can be reduced by means of fuzzification introduced in storing the results of elementary accumulation for pairs of pixels.

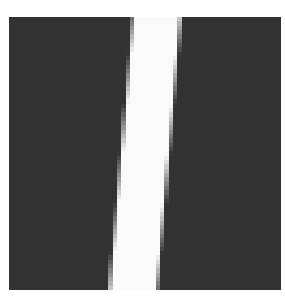

a

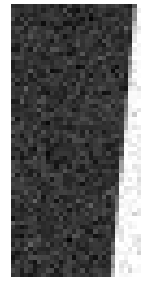

b

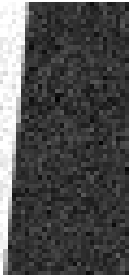

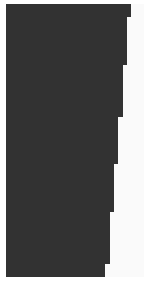

c

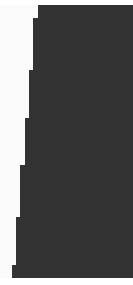

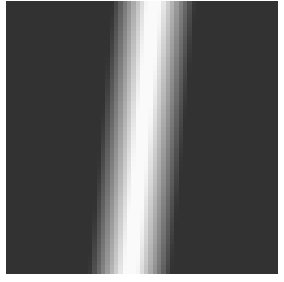

d

Fig. 3. Central windows $(63 \times 63)$ of examples of test images used in the tests. Here, line normal inclination angle is $5^{\circ}$. (a) with normal anti-aliasing; (b) anti-aliasing and noise $\sigma=15 ;(\mathbf{c})$ no anti-aliasing; (d) slope edges. 
a

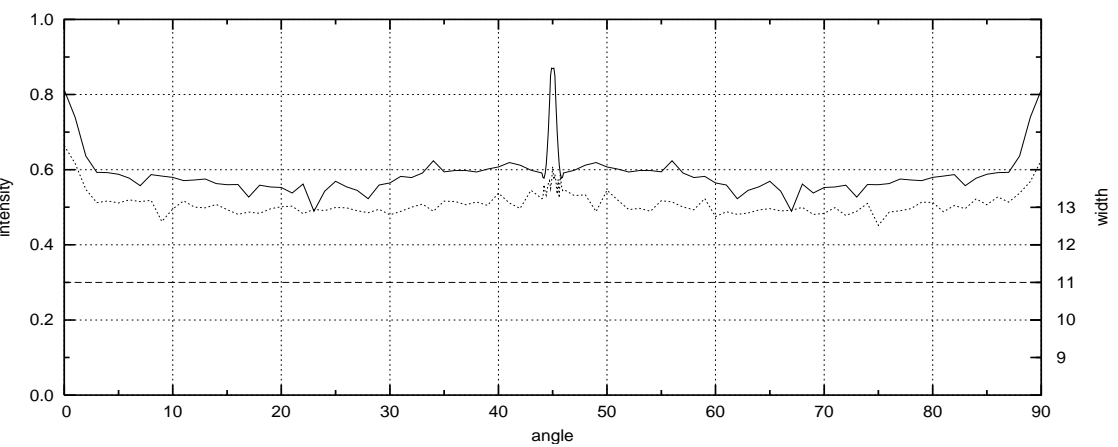

b

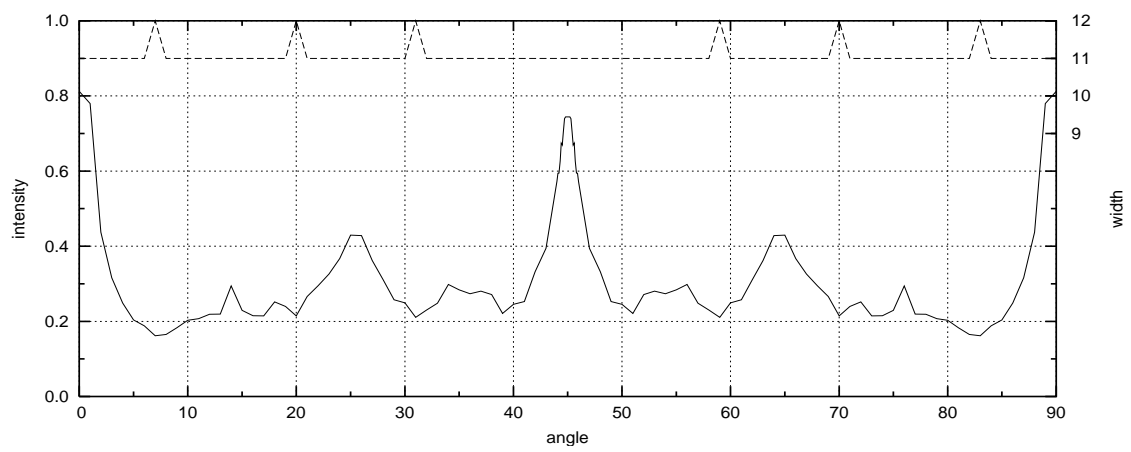

c

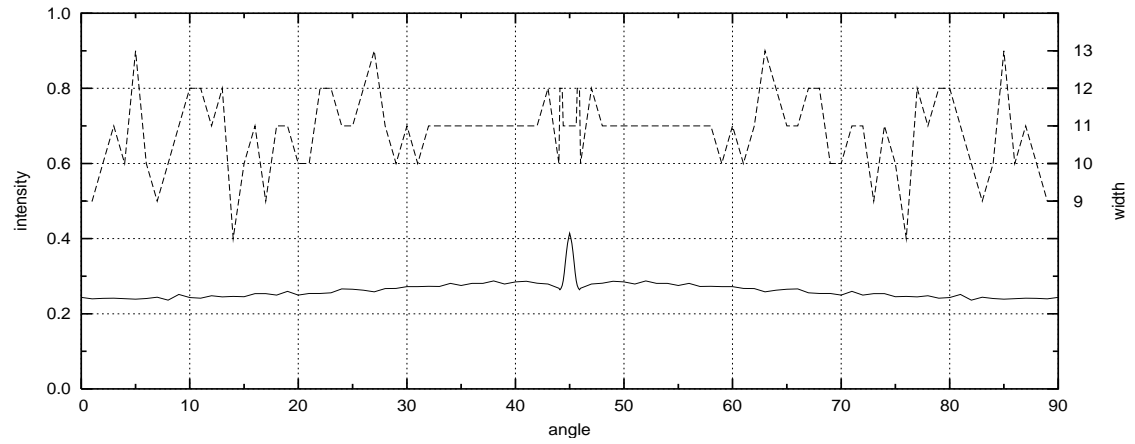

Fig. 4. Results in the central pixel of the algorithm before the modification: line intensity (solid line) and width (dashed line); (a) normal anti-aliasing, clear (solid line) and with noise (dotted line); (b) no anti-aliasing; (c) slope edges.

It is proposed to introduce a fuzzy membership function of width defined on triplets of neighbouring widths. Each elementary accumulation is performed for a given width and its two neighbouring ones. The tested membership functions were: flat function $(1,1,1)$, function $(1 / 2,1,1 / 2)$ and $(2 / 3,1,2 / 3)$. Results for the normally anti-aliased lines (Fig. 3a) are shown in Fig. 7 . The results for the function $(2 / 3,1,2 / 3)$ appeared to be the most uniform and this function has been finally chosen. 


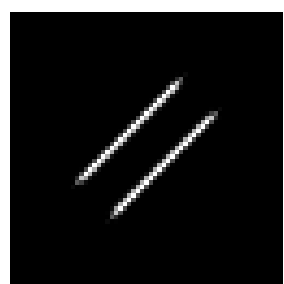

a

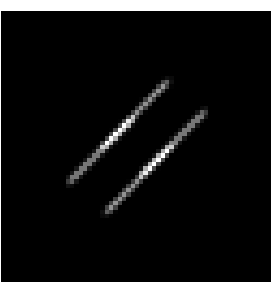

b

Fig. 5. Maps of influence of pixels on the accumulated value. Brighter pixels have larger influence. (a) for angle $45.0^{\circ}$; (b) for angle $44.2^{\circ}$ - the minimum in the graph from Fig. 4a, solid line.

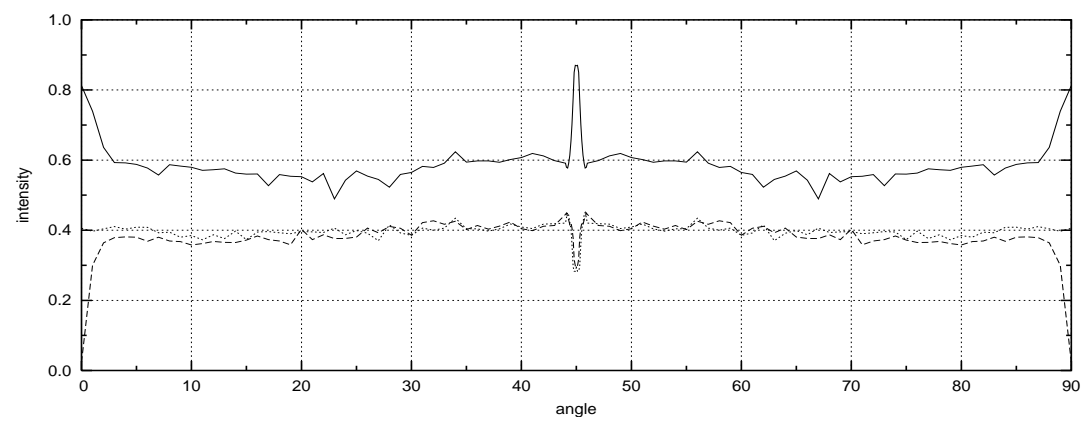

Fig. 6. Result of accumulation for three neighbouring widths: the expected width 11 (solid line), width 10 (dashed line) and width 12 (dotted line). For angles close to $0^{\circ}$ and $45^{\circ}$ less votes than usual go to widths other than that expected.

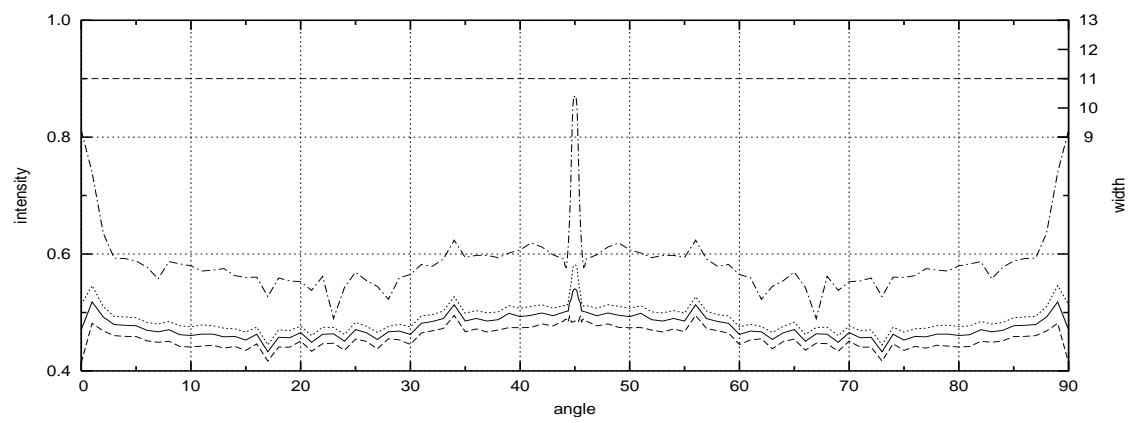

Fig. 7. Result of accumulation before (dash-dot line) and after fuzzification: with flat membership function $(1,1,1)$ (dashed line), with function $(1 / 2,1,1 / 2)$ (dotted line) and with function $(2 / 3,1,2 / 3)$ (solid line).

An example of results with and without the rotation invariance received for a test image can be seen in Fig. 8. The test image is a rosette with lines 7 pixel wide at $5^{\circ}$ angular intervals. Lines have maximum intensity 165 and have the longitudinal shape of a $\cos ^{2}$ function having maxima at a distance of 


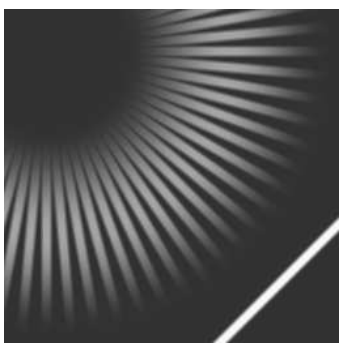

$\mathbf{a}$

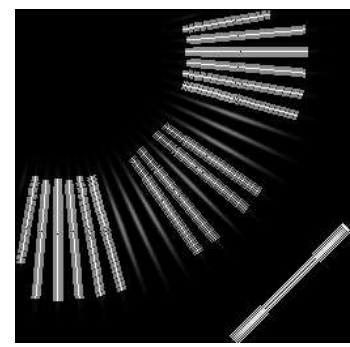

b

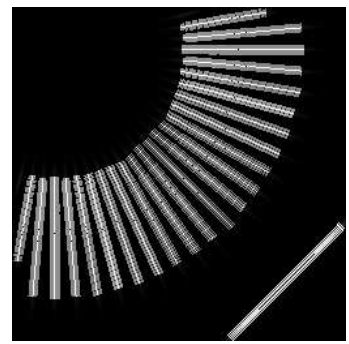

C

Fig. 8. Results for the rosette test image. (a) input image; (b) result of the directionally non-invariant algorithm; (c) result of the directionally invariant one.

140 from the centre in pixel $(32,32)$ and the period of 200 pixels. Transversal profile of lines is a result of dithering the intensity into slopes 3 pixel wide. The line in the lower right corner has intensity of 250 . This line is necessary to receive the maximum accumulated value so that the intensities of the lines in the rosette can be thresholded with respect to it.

The result of the algorithm with the rotation invariance enhanced by the fuzzification in width does not exhibit the effect of variable sensitivity to lines depending on their inclination, as the primary algorithm does.

An example for a real-life image is shown in Fig. 9. The input image is a window $250 \times 250$ starting from pixel $(3250,650)$ of the mammographic image MDB059LS.TIF from the MIAS database [4], originally $4320 \times 1600$. Calculations were made for line widths from 15 to 25 and lineness threshold coefficient 0.67 . Time of calculation was 6 and $7 \mathrm{~min}$ for the two algorithms, respectively (Pentium $1 \mathrm{Ghz}$ ).

In the result of the algorithm with the rotation invariance enhanced by the fuzzification in width the spurious lines stemming as extensions from the line in the centre were eliminated.

\section{Conclusion and suggested future work}

The scale and rotation invariance of the recently proposed line detection algorithm using the evidence accumulation principle was studied. Each of the considered features was tested on specially designed images. The modifications of the algorithm aiming at improving its invariance properties were introduced and tested on selected real-life images from the data base of mammographic images. As a result, good scale and rotation invariance properties were received. This seems to confirm the suitability of the algorithm of interest to be used in the analysis of mammographic images. The future work should go towards comparative tests of the detector against the existing detectors which use other principles $[3,5]$, with the use of real-life images. 
$\mathbf{a}$

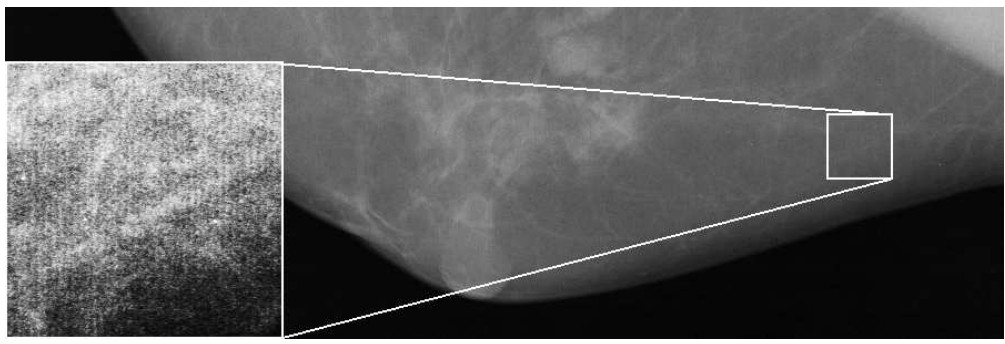

b

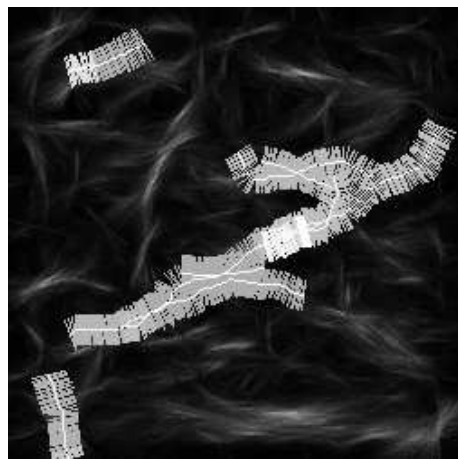

c

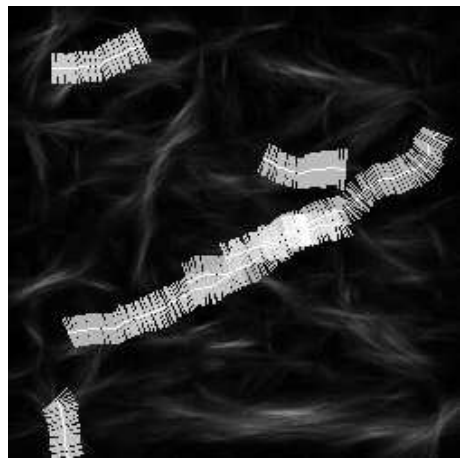

Fig. 9. Example of results for rotation invariance for a mammographic image. (a) input: window of image MDB059LS.TIF from the MIAS database; (b) accumulated lineness and line widths, directionally non-invariant algorithm; (c) the same for the directionally invariant algorithm - number of spurious lines smaller.

\section{References}

1. Chmielewski, L.: Detection of non-parametric lines by evidence accumulation: Finding blood vessels in mammograms. In Wojciechowski, K., et al., eds.: Computer Vision and Graphics: Proc. Int. Conf. Computer Vision and Graphics ICCVG 2004. Volume 32 of Computational Imaging and Vision., Warsaw, Poland, Springer Verlag, 2006 (22-24 Sep 2004) 373-380 doi:10.1007/1-4020-4179-9_54.

2. Chmielewski, L.: Specification of the evidence accumulation-based line detection algorithm. In Kurzyński, M., et al., eds.: Computer Recognition Systems: Proc. Int. Conf. CORES 2005. Volume 30 of Advances in Soft Computing., Rydzyna, Poland, Springer Verlag (22-25 May 2005) 355-362 doi : 10.1007/3-540-32390-2_41.

3. Zwiggelaar, R., Astley, S., Boggis, C., Taylor, C.: Linear structures in mammographic images: detection and classification. IEEE Trans. Signal Procesing 23(9) (Sep 2004) 1077-1086 doi:10.1109/TMI.2004.828675.

4. Suckling, J., Parker, J., Dance, D., et al.: The Mammographic Images Analysis Society digital mammogram database. In Gale, A., Astley, S., Dance, D., Cairns, A., eds.: Digital Mammography. Volume 1069 of Exerpta Medica International Congress Series., Elsevier (1994) 375-378 www.wiau.man.ac.uk/services/MIAS/; later moved to peipa.essex.ac.uk/info/mias.html. 
Scale and Rotation Invariance of the Evidence Accumulation-Based...

5. Zwiggelaar, R., Boggis, C.: Classification of linear structures in mammographic images. In: Proc. Conf. Medical Image Understanding and Analysis MIUA 2001, Birmingham, UK (16-17 Jul 2001) 